\title{
Predictive K-PLSR Myocardial Contractility Modeling with Phase Contrast MR Velocity Mapping
}

\author{
Su-Lin Lee, Qian Wu, Andrew Huntbatch, and Guang-Zhong Yang \\ Institute of Biomedical Engineering, Imperial College London, UK \\ \{su-lin.lee, q.wu, a.huntbatch, g.z.yang\}@imperial.ac.uk
}

\begin{abstract}
With the increasing versatility of CMR, further understanding of intrinsic contractility of the myocardium can be achieved by performing subject-specific modeling by integrating structural and functional information available. The recent introduction of the virtual tagging framework allows for visualization of the localized deformation of the myocardium based on phase contrast myocardial velocity mapping. The purpose of this study is to examine the use of a non-linear, Kernel-Partial Least Squares Regression (K-PLSR) predictive motion modeling scheme for the virtual tagging framework. The method allows for the derivation of a compact non-linear deformation model such that the entire deformation field can be predicted by a limited number of control points. When applied to virtual tagging, the technique can be used to predictively guide the mesh refinement based on the motion of the coarse grid, thus greatly reducing the search space and increasing the convergence speed of the algorithm. The effectiveness and numerical accuracy of the proposed technique are assessed with both numerically simulated data sets and in vivo phase contrast CMR velocity mapping from a group of 7 subjects. The technique presented has a distinct advantage over the conventional mesh refinement scheme and brings CMR myocardial contractility analysis closer to routine clinical practice.
\end{abstract}

\section{Introduction}

Heart failure due to coronary artery disease has considerable morbidity and poor prognosis. An understanding of the underlying mechanics governing myocardial contraction is a prerequisite for interpreting and predicting changes induced by heart disease. Gross changes in contractile behavior of the myocardium are readily detected with existing techniques. For more subtle changes during early stages of cardiac dysfunction, however, it requires a sensitive method for measuring, as well as a precise criterion for quantifying, normal and impaired myocardial function. For this purpose, Cardiovascular Magnetic Resonance (CMR) imaging has taken a key role in investigating regional contractile function as it provides a non-invasive and relatively easy way of assessing the intramural motion of the myocardium.

Thus far, the main techniques of CMR in measuring myocardial contraction include tagging, displacement encoding (DENSE), and myocardial velocity mapping. In vivo studies using tagging and velocity mapping have demonstrated the value of 
CMR in illustrating the direct link between diseases such as ischemic heart disease and cardiomyopathies with intrinsic myocardial contractility. Diffusion tensor imaging augmented with strain rate from velocity mapping has also been shown to play an important role in elucidating the relationship between fiber orientation and fiber shortening.

With the increasing versatility of CMR, a natural step towards the further understanding of intrinsic myocardial contractility is to perform subject-specific biomechanical modeling by effectively integrating the structural and functional information available. To this end, 3D modeling of the heart with the use of different deformation tracking methods has been investigated. Currently, most of the work on tracking motion of the heart has incorporated MR tagging [1]. Although tagging has been shown to be a reliable means of determining the intramural motion of the myocardium, the issue of tag fading and the complicated post-processing steps involved in deriving strain distribution have imposed significant limitations on the technique. There has also been much work on biomechanical modeling of the heart, incorporating parameters such as material properties and fiber orientation. Biomechanical modeling, however, requires extensive a priori data that is difficult to obtain for each subject. It is usual for a general model of the heart to be developed which is then mapped to each subject.

The alternative of myocardial velocity imaging has traditionally been riddled with problems such as blood flow artifact, limitations on velocity sensitivity, and low SNR. However, recent work on improved pulse sequence design with blood saturation to limit the blood flow artifact has shown great promise of the technique in providing reliable, detailed myocardial contractility information that can be directly used for cardiac modeling [2]. Previous research has shown that it is possible to use a virtual tagging framework to derive strain distribution from the myocardial velocity data [3]. The concept of virtual tagging is based on the superimposition of an artificial tag pattern onto the CMR velocity data and observing its subsequent deformation. At a given interval of the cardiac cycle, if the deformation of the virtual tags reflects the true motion of the myocardium, the associated displacement, and hence the velocity distribution, should be identical to the directly measured CMR velocity data in a leastmean-squares sense. With this framework, the deformation of the myocardium is controlled by the underlying velocity vectors, and therefore it removes the need for $a$ priori information for myocardial contractility analysis. While the technique is promising, it is computationally demanding.

The purpose of this study is to examine the use of a non-linear, Kernel-Partial Least Squares Regression (K-PLSR) predictive motion modeling scheme for the virtual tagging framework. The basic idea of the technique is to use K-PLSR to derive a compact non-linear deformation model such that the entire deformation field can be predicted by a limited number of control points. When applied to virtual tagging based on myocardial velocity mapping, the technique can be used to predictively guide the mesh refinement based on the motion of the coarse grid, thus greatly reducing the search space and increasing the convergence speed of the algorithm. For assessing the effectiveness and numerical accuracy of the proposed technique, both numerically simulated data sets and in vivo phase contrast CMR velocity mapping of the left ventricle are used. 


\section{Methods}

\subsection{K-PLSR Predictive Model for Virtual Tagging}

The Virtual Tagging framework [3] combines the advantages of both the MR tagging and velocity mapping techniques. With this technique, a superimposition of an artificial tag pattern onto the CMR velocity data is the basis of this framework; the tag pattern can take any desired configuration. In this work, the 3D tag configuration is defined by the subdivision solids volume model. The deformation of the virtual tags is controlled by the underlying velocity data and provides easy visualization and direct assessment of myocardial motion. During optimization, the objective function directly minimizes the difference between the velocity vectors and the application of a mass conservation constraint ensures physically meaningful results. This results in the following cost function:

$$
E=\sum_{i}\left|\Delta \vec{v}_{i}\right|^{2} \Delta t^{2}+\lambda \sum_{i}\left|\xi_{i}-1\right| S_{i}
$$

where $\vec{v}_{i}$ is the difference between the measured velocity and the virtual tagging simulation velocity, $\Delta t$ is the time interval between consecutive timeframes, $\xi_{i}$ is the ratio of volumes between the measured and virtual tagging simulation elements, and $S_{i}$ is the surface area of each element. In Eq. (1), $\lambda$ is a weighting factor that controls how much the virtual tags deform, causing either the velocity error measure or the volume control to have a more significant impact on the deformation.

One of the significant problems of virtual tagging is its computational complexity. To circumvent this problem, predictive motion modeling based on non-linear regression is applied. Regression analysis is a statistical tool that examines the dependence of a number of response variables on a number of predictors. K-PLSR is a technique to construct a nonlinear regression model in high dimensional feature spaces. This technique is an extension of PLSR, which can extract correlation between input and output data that is highly collinear - making it ideal for problems inappropriate for multi-linear or principal components regression. In PLSR, a simultaneous decomposition of $\mathbf{X}$ and $\mathbf{Y}$, the predictors and response variables respectively, are performed, i.e.,

$$
\begin{aligned}
& \mathbf{X}=\mathbf{T} \mathbf{P}^{\mathrm{T}}+\mathbf{E} \\
& \mathbf{Y}=\mathbf{U} \mathbf{Q}^{\mathrm{T}}+\mathbf{F}
\end{aligned}
$$

where $\mathbf{T}$ and $\mathbf{U}$ are extracted score vectors, $\mathbf{P}$ and $\mathbf{Q}$ are loading matrices, and $\mathbf{E}$ and $\mathbf{F}$ are residuals, to give the maximal covariance of $\mathbf{X}$ and $\mathbf{Y}$. The response variables are predicted as:

$$
\hat{\mathbf{Y}}=\mathbf{T D Q}^{\mathrm{T}}
$$

where $\mathbf{D}$ is a diagonal matrix containing the regression weights.

For describing non-linear motion models, K-PLSR can be used, with which the input variables are first mapped by a function $\Phi$ to a feature space where a linear PLSR is performed [4]. In the original space, this results in a nonlinear regression 
model. In this study, as we are dealing with more observed variables than measured objects, the kernel Gram matrix $\mathbf{K}=\Phi \Phi^{\mathrm{T}}$ between all mapped input data points $\left\{\Phi\left(\mathbf{x}_{i}\right)\right\}_{i=1}^{N}$ is calculated as:

$$
\mathbf{K}=\left[\begin{array}{cccc}
K\left(x_{1}, x_{1}\right) & K\left(x_{1}, x_{2}\right) & \cdots & K\left(x_{1}, x_{N}\right) \\
K\left(x_{2}, x_{1}\right) & K\left(x_{2}, x_{2}\right) & \cdots & K\left(x_{2}, x_{N}\right) \\
\vdots & \vdots & \ddots & \vdots \\
K\left(x_{N}, x_{1}\right) & K\left(x_{N}, x_{2}\right) & \cdots & K\left(x_{N}, x_{N}\right)
\end{array}\right]
$$

where each element of the matrix is a calculation of a kernel function $K\left(x_{1}, x_{2}\right)$ based on Mercer's theorem [5]. For this application, a Gaussian kernel

$$
K(x, y)=e^{-\left(\frac{\|x-y\|^{2}}{d}\right)}
$$

is used, where $d$ is the width of the Gaussian function. While it is possible to use a single value of $d$ for each left ventricle model, we examine the distribution of $d$ across the left ventricle for optimal prediction. Predictions are made by:

$$
\hat{\mathbf{Y}}_{\mathrm{t}}=\mathbf{F}_{\mathrm{t}} \mathbf{B}=\mathbf{K}_{\mathrm{t}} \mathbf{U}\left(\mathbf{T}^{\mathrm{T}} \mathbf{K U}\right)^{-1} \mathbf{T}^{\mathrm{T}} \mathbf{Y}
$$

where $\mathbf{K}_{\mathbf{t}}$ is the "test" Gram matrix whose elements are calculated by both the testing and training points. A modification of the NIPALS algorithm is used to calculate the K-PLSR [6]. In our application, $\mathbf{X}$ and $\mathbf{Y}$ are the coarse mesh points and fine mesh points, respectively, describing the myocardium of the left ventricle.

\subsection{Data Acquisition and Model Creation}

For validating the proposed predictive modeling scheme, short-axis velocity mapping images of the heart were acquired from 7 normal subjects using a gradient-echo phase-contrast protocol $(\mathrm{TR}=53 \mathrm{~ms}, \mathrm{TE}=7.1 \mathrm{~ms}$, in-plane pixel resolution $=$ $1.17 \times 1.17 \mathrm{~mm}, \mathrm{FOV}=30 \times 30 \mathrm{~cm}, \mathrm{VENC}=-15$ to $+15 \mathrm{~cm} / \mathrm{s}$ ) on a $1.5 \mathrm{~T}$ Siemens Sonata MRI scanner. The sequence consisted of specially designed black-blood RF pulse being applied every other time frame followed by the imaging pulse. It also incorporated a $k$-space view-sharing scheme to reduce the total scan time needed, hence allowing one reference image and three orthogonally encoded velocity images to be acquired. Diaphragmatic navigator echoes were implemented which permits free-breathing data acquisition and ensures geometrical and functional consistency of the 3D cine myocardial velocity data. A total of 12 to 14 short axis slices were obtained for each subject with 10 to 17 timeframes spanning the entire cardiac cycle. Both a rigid body motion correction [7] and a Total Variational (TV) restoration technique [5] were applied to restore the data. The TV technique optimizes the variance of the image and constrains the total amount of variance removed from the image to be equal to the estimated variance of the noise component. 
For the 7 subjects studied, the epicardial and endocardial borders of the left ventricle were semi-automatically segmented using CMRtools (CVIS, London, UK) from the magnitude images, from which the corresponding velocity maps were extracted. The resultant surface mesh was used to build a volumetric model of hexahedral elements. To generate elements of sufficient size for a smoother mesh, the elements were divided using subdivision solids [8], an extension of the subdivision surfaces refinement rules developed by Catmull and Clark [9]. During this division, each hexahedral element was subdivided into a further eight elements. Under the existing virtual tagging framework, the refinement of the mesh is usually performed by coarse-to-fine mesh propagation. Linear interpolation is first used to define the initial position of the finer mesh control points, followed by locally searching for the optimal mesh control points by minimizing Eq. (1). In the presence of large, nonlinear deformation, the initial control points defined by interpolation are usually far away from the desired location, leading to poor convergence of the optimization process due to local minima. With the proposed K-PLSR predictive motion modeling, the refined mesh location is much closer to the desired configuration as dictated by the underlying myocardial velocity data, and thus the convergence of the algorithm is greatly improved.

In order to assess the performance of the proposed algorithm with known ground truth data, numerical simulation representing a twisting cube and an artificial left ventricle was also developed. The simulated left ventricle exhibits the properties inherent in a normal left ventricle with radial thickening and longitudinal shortening. Furthermore, the volume of the simulated set obeys the mass conservation rule, similar to the properties of cardiac muscle. A leave-one-out analysis was performed on the simulated and in vivo left ventricles, with each training set consisting of the entire series save one, and the prediction performed on the left ventricle left out.

\section{Results}

Fig. 1 illustrates the result of applying the proposed K-PLSR motion modeling scheme to the twisting cube data set. During simulation, the coarse mesh points were used as input and fine mesh points as output. It is evident that despite the presence of large non-linear distortion, the proposed K-PLSR method is able to predict the distortions involved. With K-PLSR, the error involved is represented in a false colormap in Fig. 1(b). The average error involved was $3.6 \times 10^{-5} \pm 2.4 \times 10^{-5}$ (normalized to the width of the cube). This translated to less than $2 \%$ of a voxel width when the object is represented with a $512 \times 512 \times 512$ grid. To facilitate the visualization of the error distribution, the color map in Fig. 1(b) was normalized to the scale of the average error, despite the fact that it is negligible.

For the simulated left ventricular data set, the graph in Fig. 2(b) shows the effect of the number of latent vectors used for prediction on the simulated left ventricle model. The maximum average error across the cycle is approximately $0.08 \mathrm{~mm}$. The same analysis performed with linear PLSR showed a maximum average error of $0.19 \mathrm{~mm}$, highlighting the necessity of a nonlinear regression. The simulated data set has a $75 \mathrm{~mm}$ diameter and is $64 \mathrm{~mm}$ in length. 


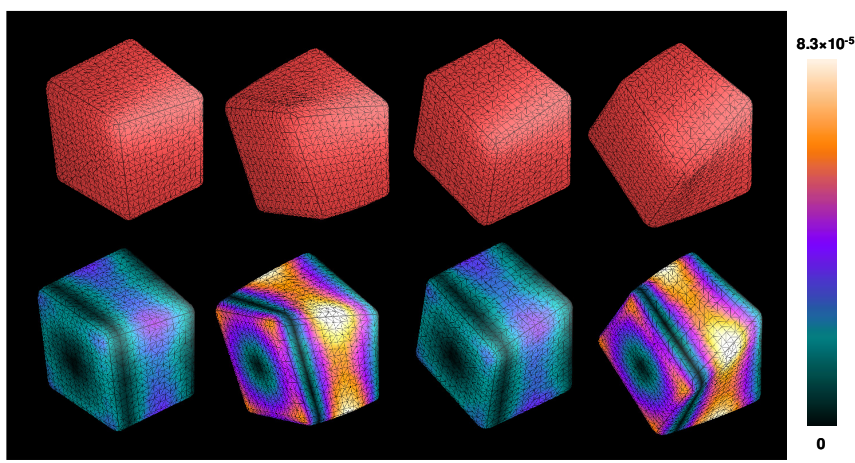

Fig. 1. An example of the predictive properties of the proposed K-PLSR modeling technique. From a training set consisting of a series of distorted cubic shapes, predictions were made on intermediate shapes (top). The associated prediction error between the predicted shapes and the ground truth data are shown (bottom). The maximum color scale corresponds to $8.3 \times 10^{-5}$ normalized to the width of the cube.

(a)

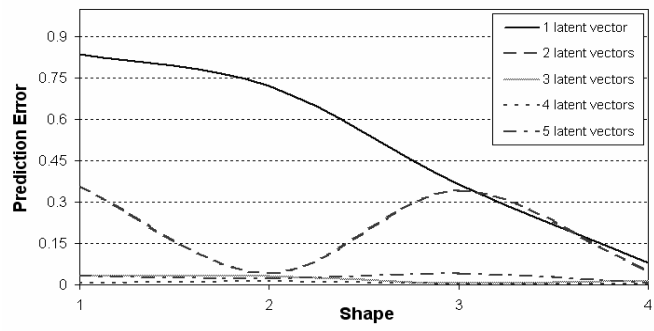

(b)

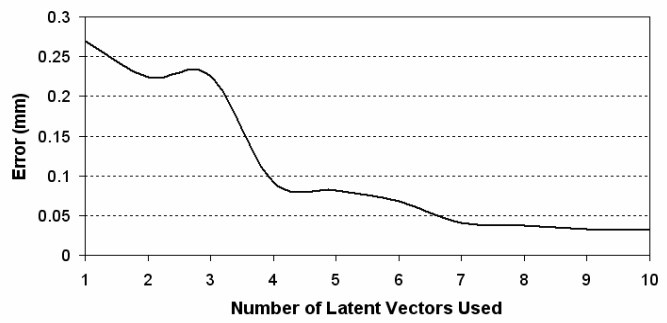

Fig. 2. (a) The effect of the number of latent vectors used for the K-PLSR model on the prediction accuracy on the distorted cubic shapes. (b) The mean prediction error on the leaveone-out analysis of the simulated left ventricle versus the number of latent vectors used.

The proposed technique was subsequently applied to the myocardial velocity data for the 7 subjects studied. Fig. 3(top) provides an example of the short axis image at each phase of the cardiac cycle and the corresponding myocardial velocity components along the $x, y$, and $z$ axes. Fig. 3(middle) shows the virtual tagging deformation of that in vivo left ventricle from diastole to systole, with the longitudinal strain overlaid. In Fig. 3(bottom), plots of the error at each node of the predicted left ventricle shape are shown for the example in vivo set. 


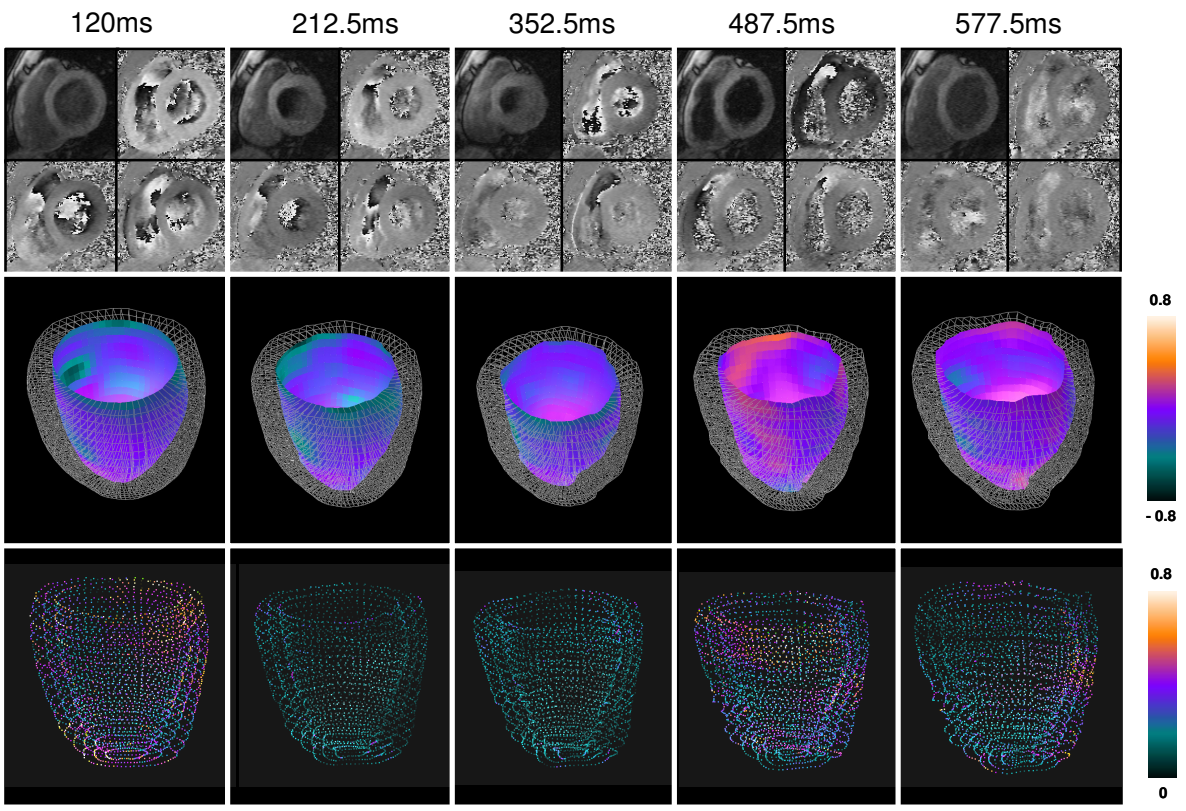

Fig. 3. (top) Short axis slices of the myocardium at five selected phases of the cardiac cycle, and the corresponding myocardial velocity components along the $x, y$ and $z$ directions. (middle) The longitudinal strain as derived from the virtual tagging framework by using the proposed predictive motion modeling scheme, and the corresponding prediction error at each node in $\mathrm{mm}$ (bottom).

Table 1. The mean, maximum and standard deviation of the prediction error for the 7 subjects studied by using the proposed K-PLSR predictive motion modeling scheme

\begin{tabular}{cccc}
\hline Subject & Mean Error $(\mathbf{m m})$ & Max Error $(\mathbf{m m})$ & Std Error $(\mathbf{m m})$ \\
\hline 1 & 0.119 & 0.585 & 0.071 \\
2 & 0.153 & 0.748 & 0.259 \\
3 & 0.127 & 1.213 & 0.076 \\
4 & 0.159 & 0.832 & 0.006 \\
5 & 0.489 & 2.493 & 0.282 \\
6 & 0.346 & 1.978 & 0.197 \\
7 & 0.187 & 1.035 & 0.110 \\
\hline
\end{tabular}

Finally, Table 1 summarizes the mean, maximum, and standard deviation of the errors of the proposed method to the 7 subjects studied. It is evident that the predictions are close to the ground truth with mean errors across all subjects less than $1 \mathrm{~mm}$. Finding a single optimal kernel parameter and the predicted nodes for a single shape takes on average 2 minutes. This time will increase should a distribution of optimal kernel parameters be required but this is still significantly less time than that required by virtual tagging alone. 


\section{Discussions and Conclusions}

In summary, we have presented a predictive framework for the examination of myocardial contractility by combining the advantages of both K-PLSR and virtual tagging frameworks. The main strengths of the virtual tagging framework remain in the flexibility of the technique in establishing material correspondence of the myocardium across the entire cardiac cycle. The results have shown that the proposed non-linear predictive model is a promising technique for improving the performance of the virtual tagging framework. The use of K-PLSR allows the derivation of a compact non-linear deformation model such that the entire deformation field can be predicted by a limited number of control points. The technique can be used to predictively guide the mesh refinement based on the motion of the coarse grid, thus greatly reducing the search space and increasing the convergence speed of the algorithm. The technique presented has a distinct advantage over the conventional mesh refinement scheme and brings the idea of CMR myocardial contractility analysis closer to routine clinical practice.

Acknowledgments. The authors would like to thank Karim Lekadir for discussions on statistical modeling and Prof. David Firmin for his help with image acquisition.

\section{References}

[1] Axel, L., Dougherty, L.: MR imaging of motion with spatial modulation of magnetization. Radiology 171(3), 841-845 (1989)

[2] Jung, B., Schneider, B., Markl, M., et al.: Measurement of left ventricular velocities: phase contrast MRI velocity mapping versus tissue-doppler-ultrasound in healthy volunteers. J. Cardiovasc. Magn. Reson. 6(4), 777-783 (2004)

[3] Masood, S., Gao, J., Yang, G.-Z.: Virtual tagging: numerical considerations and phantom validation. IEEE Transactions on Medical Imaging 21(9), 1123-1131 (2002)

[4] Ablitt, N.A., Jianxin, G., Keegan, J., et al.: Predictive cardiac motion modeling and correction with partial least squares regression. IEEE Transactions on Medical Imaging 23(10), 1315-1324 (2004)

[5] Ng, Y.-H.P., Yang, G.-Z.: Vector-valued image restoration with applications to magnetic resonance velocity imaging. Journal of WSCG 11(1) (2003)

[6] Rosipal, R., Trejo, L.J.: Kernel Partial Least Squares Regression in Reproducing Kernel Hilbert Space. Journal of Machine Learning Research 2, 97-123 (2002)

[7] Arai, A.E., Gaitha III, C.C., Epstein, F.H., et al.: Myocardial velocity gradient imaging by phase contrast MRI with application to regional function in myocardial ischemia. Magnetic Resonance in Medicine 42(1), 98-109 (1999)

[8] MacCracken, R., Joy, K.I.: Free-Form Deformations With Lattices of Arbitrary Topology. In: the 23rd Annual Conference on Computer Graphics and Interactive Techniques, pp. 181-188 (1996)

[9] Catmull, E., Clark, J.: Recursively generated B-spline surfaces on arbitrary topological meshes. Computer-Aided Design 10(6), 350-355 (1978) 